\title{
Analysis of roughness of Cs surfaces via evaluation of the autocorrelation function
}

\author{
A. Fubel ${ }^{\mathrm{a}, *}$, M. Zech ${ }^{\mathrm{a}}$, P. Leiderer ${ }^{\mathrm{a}}$, J. Klier ${ }^{\mathrm{a}}$, V. Shikin ${ }^{\mathrm{b}}$ \\ a Department of Physics, University of Konstanz, D-78457 Konstanz, Germany \\ b ISSP, 142432 Chernogolovka, Moscow District, Russia
}

Received 20 October 2006; accepted for publication 19 January 2007

Available online 2 February 2007

\begin{abstract}
At low temperature prepared quench-condensed Cs surfaces are analysed on a nanometer scale via scanning tunneling microscopy. The analysis of surface roughness is presented with the help of the evaluation of their autocorrelation function. In order to extract the correct autocorrelation function we present the requirement regarding the scan resolution of scanning probe microscopy (SPM) images in general. This is supported by a 'numerical experiment'. Furthermore, we present some methods of deducing higher orders of autocorrelation lengths, which are needed to evaluate SPM images with non-random distribution of roughness amplitudes. These characteristic values of the autocorrelation function could play the key role in further statistical calculations, e.g., on how surface roughness alters the wetting behaviour of liquid helium adsorbed on the cesium surfaces.
\end{abstract}

(C) 2007 Elsevier B.V. All rights reserved.

Keywords: Scanning tunneling microscopy; Surface structure, morphology, roughness, and topography; Surface diffusion; Surface roughening; Computer simulations; Alkali metals; Polycrystalline surfaces; Polycrystalline thin films; Metallic films

\section{Introduction}

The characterisation of cesium surfaces is important to understand the wetting behaviour of liquid helium on Cs [1], since this is, in principle, an ideal model system for wetting studies [2]. However, Cs is highly reactive, hence it is difficult to access with probing instruments. Despite these difficulties it was possible to investigate, on a sub-micron lengthscale, the surface of in situ prepared Cs films via scanning tunneling microscopy (STM) at low temperatures [3].

In general, surface roughness is an inevitable property of nearly all solid substrates (except cleaved substrates under proper environmental conditions [4]) and so usually plays an important role in wetting phenomena and thin film physics. This applies, for example, for wetting experiments where the contact angle hysteresis as function of the rough-

\footnotetext{
* Corresponding author.

E-mail address: armin.fubel@uni-konstanz.de (A. Fubel).
}

ness of nano-structured surfaces are studied [5], fluid dynamics measurements where the influence of roughness on the no-slip boundary condition is analysed [6], theoretical investigation of roughness-induced wetting [7], increased thickness of liquid films in the presence of roughness [8], scattering experiments from non-perfect liquidvapour interfaces (see e.g. Ref. [9]), roughness limited mobility measurements in low dimensional charged systems (see e.g. Refs. [10-12]), thermal fluctuation physics (see e.g. Ref. [13]), and so on. A summary of the common approaches of quantitative descriptions of surface roughnesses is given in Ref. [14].

For a surface analysis we use two basic characteristics of a one-dimensional roughness profile $Z(x)$, where $Z$ is the height over a fixed zero level (usually the mean $Z$-value) at the position $x$. One of them is the distribution function $W(Z, \Delta)$ of the roughness amplitude, i.e.,

$$
\int_{-\infty}^{+\infty} W(Z, \Delta) \mathrm{d} Z=1, \quad\left\langle Z^{2}\right\rangle=\Delta^{2}
$$


For example, for a random roughness $W(Z, \Delta)$ is a Gauss distribution, where $\Delta$ is the standard deviation with respect to $Z$. The second characteristic is the autocorrelation function $C(l)$, where

$C\left(x-x_{1}\right)=\left\langle Z(x) Z\left(x_{1}\right)\right\rangle \quad$ with $C(0) \simeq \Delta^{2}$ and $l=x-x_{1}$.

As the mean values of the profiles or images treated in this paper are equal to zero $C(l)$ could also be called autocovariance function [14]. A purely random roughness, in sense of the spatial distribution of surface heights, leads to an autocovariance function with the shape of a delta function centered at the origin [14,15]. Definitions (1) and (2) correspond to the so-called 'normal' randomness, where the roughness properties are translationally invariant. To be as simple as possible we use 'one-dimensional' definitions.

The aim of this paper is the analysis of STM measurements concerning the roughness properties, as defined in Eqs. (1) and (2), of in situ and at low temperature prepared cesium surfaces. We complement the experimental data by methodical considerations which help to classify scanning probe microscopy (SPM) measurements. In most cases of autocorrelation analysis so far only the initial portion of the autocorrelation function is evaluated, e.g., fitted [16]. We investigate the further development of the curve and extract, for example, higher orders of correlation lengths. These efforts are needed to investigate SPM data which have a non-random distribution of roughness amplitudes, as many images of scanned surfaces show the existence of more than one correlation length. Under these points of view we structure the paper as follows:

In Section 2 we discuss how accurate the profile measurements have to be in order to extract the correct correlation length $\gamma$ from the experimental data. Intuitively, the measuring distance should be less than the correlation length. But in the beginning of the measurements there is no information about the real scale of $\gamma$. Therefore it is useful to have a reliable method for optimising the measurement regarding the necessary resolution. The development of these considerations helps to formulate details of fitting STM data in presence of several correlation lengths.

In Section 3 we apply the obtained methodic rule to the real surface data from the STM images of quench-condensed cesium films. The analysis of these data shows that a formalism with just one correlation length is not enough to explain the details of STM measurements. Hence, we need a more complicated scenario for a better understanding of the qualitative progression of the curve of the autocorrelation function.

\section{Optimisation of scanning probe microscopy measurements}

(A): As it was indicated in the introduction we will support the correlation analysis of STM data with some kind of numerical 'experiment', and so to illustrate the optimal conditions for extracting the autocorrelation function. To motivate the following procedure we show in Fig. 1 an experimental STM image of a cesium surface, which will be analysed in Section 3, and a profile $Z(x)$ corresponding to the position marked with the white line.

For a systematic study we replace the profile with a simple analytic definable function which has a random component, though: a so-called random telegraph function. The simplest version consists of only two amplitudes: $Z_{+}=$ +1 and $Z_{-}=-1$. The position where the sign changes are determined by a process described below. For a better comparability with an experimental profile several random telegraph functions could be summed up to get many different amplitudes. However, the simplest version is sufficient for a correlation analysis and our numerical 'experiment'. An example of a possible $Z(x)$ for the simplest case is shown in Fig. 2a:

$Z(x)=(-1)^{n(x)}$,

where $n(x)$ is created in the following way: We generate $N=50$ random real numbers within the interval $s=50$ unit lengths (UL), which is the length of the investigated profile. These random numbers are sorted by size. Hence, we form a discrete Markov chain which contains correlations $[17,18]$. The sorted numbers are taken as the $x$-values where $n(x)$ increases by one, see for example Fig. $2 \mathrm{~b}$, and where, according to Eq. (3), $Z(x)$ changes its sign, see Fig. 2a.

So $N$ is the total number of points, where the profile sign changes, and $v=N / s$ is the value of the average slope of $n(x)$ and thus the average number of changes in sign of the profile per UL. We will use this parameter later on. To improve the statistical distribution we take 40 profiles with the same parameters, but different random numbers,
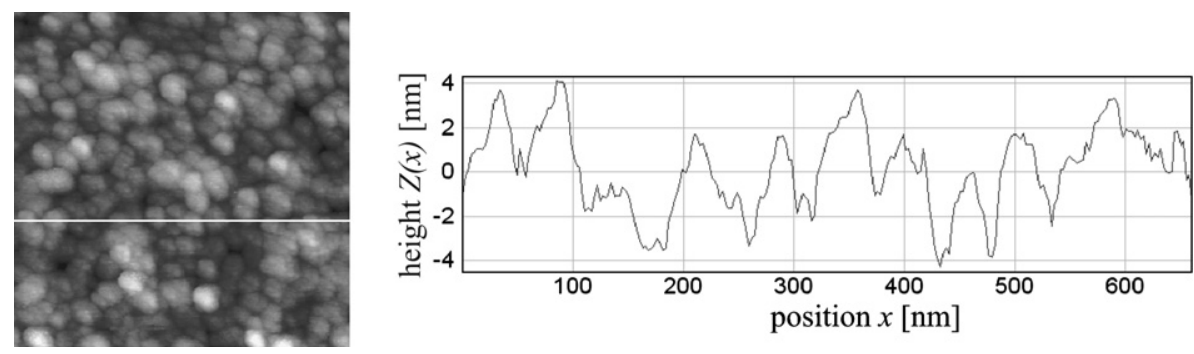

Fig. 1. STM image of a Cs surface with a side length of $s=660 \mathrm{~nm}$. The white line indicates the position of the profile $Z(x)$ shown on the right. The profile has a height-to-length ratio of 1:20. The line consists of 256 sample points which hence have a distance of about $2.6 \mathrm{~nm}$. 

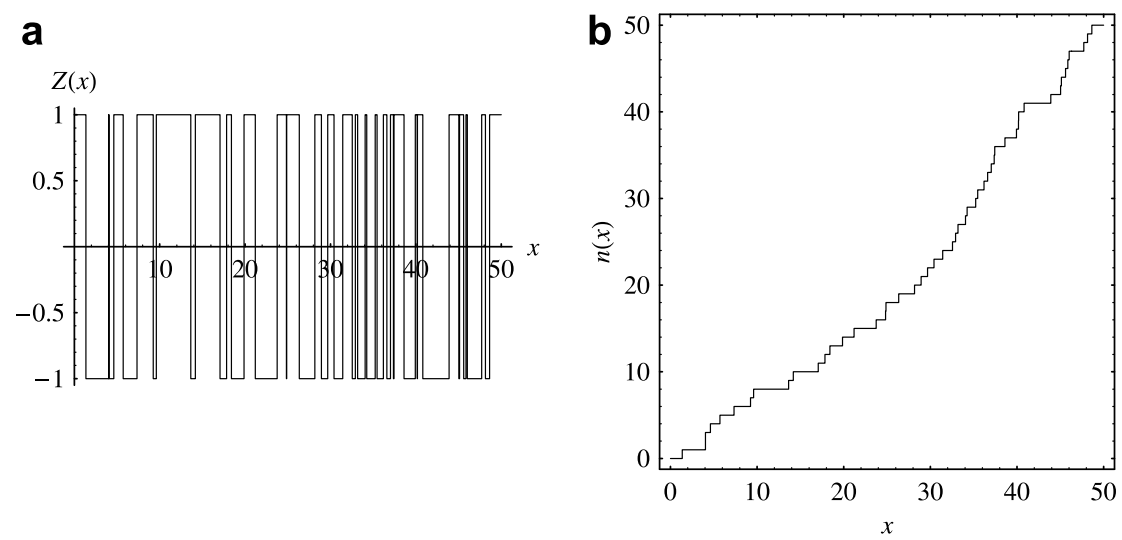

Fig. 2. (a) An example of the so-called telegraph function $Z(x)$, see Eq. (3), with the $n(x)$ as shown in (b). (b) An example of $n(x)$. The $x$-values are randomised and sorted by size (Markov process [17]).

and we will average the resulting autocorrelation functions of each profile.

The autocorrelation function of $Z(x)$ is analytically established [18]:

$\left\langle Z(x) Z\left(x_{1}\right)\right\rangle=\Delta^{2} \exp \left\{-\frac{1}{\gamma}\left(x-x_{1}\right)\right\}$

where $x \geqslant x_{1}$. In these terms the correlation length $\gamma$ is that $l$-value where $C(l)$ has decreased to $1 / e(\approx 37 \%)$ and $\Delta$ is the value of $\sqrt{\left.C(l)\right|_{l \rightarrow 0}}$, which conforms the standard deviation from Definitions (1). Hence, we have $\gamma=0.5 \mathrm{UL}$ and $\Delta=1$.

Now we investigate, on the basis of our test profile $Z(x)$, how dense a set of selective points has to be in order to extract with sufficient accuracy the autocorrelation function from the profile. We fix a set of selective points

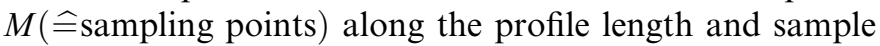
$Z(x)$ from Eq. (3) at these points. So we get a 'virtually measured' profile $Z^{\prime}(x)$, which is used for the calculation of the correlation length, as explained below. $M$ corresponds to the set resolution of a scanned line of a SPM image. This sampling accuracy is given in a more general way by the sampling points per UL: $m(=M / s)$ divided by $v$. In the following we will use the three ratios (with $v=1$ ):

$m / v=10, \quad M=500$,

$m / v=1, \quad M=50$,

$m / v=0.1, \quad M=5$.
Fig. 3a and $\mathrm{b}$ show the resulting profiles $Z^{\prime}(x)$ for the cases (5) and (6). In Fig. 3a the original profile is reflected quite well, whereas in Fig. $3 \mathrm{~b}$ much less details are shown. The third case describes the approximated profile with only six points (not shown), which are obviously too few to show any structure and just lead to an almost flat surface.

By definition, $C(l)$ can be extracted from the 'measured' data $Z^{\prime}(x)$ using the corresponding Fourier analysis:

$$
\begin{aligned}
& Z^{\prime}(q)=\frac{1}{2 \pi} \int_{-\infty}^{+\infty} Z^{\prime}(x) \mathrm{e}^{\mathrm{i} q x} \mathrm{~d} x, \\
& C(l)=\int_{-\infty}^{+\infty}\left(Z^{\prime}(q)\right)^{2} \mathrm{e}^{-\mathrm{i} q l} \mathrm{~d} q .
\end{aligned}
$$

For a $C(l)$, which fulfils the requirements as described in the introduction, the distance between the 'numerical' points has to be small compared to the correlation length. If we use the ratio of parameters from Eqs. (5)-(7), i.e., a finite number of sampling points, then the integrals (8) and (9) will exchange into discrete sums with the lower and upper limits $-M / 2$ and $+M / 2$, respectively. In this way, we receive autocorrelation functions as presented in Figs. 4 and 5. In these diagrams we can see that for the case (5) the autocorrelation function has the expected structure, since the exponential decay can be recognised. So there are enough points between zero and $\gamma$ (dotted line in the inset of Fig. 4 and in Fig. 5). Theoretically the autocorrelation function should exponentially approach zero. The limited
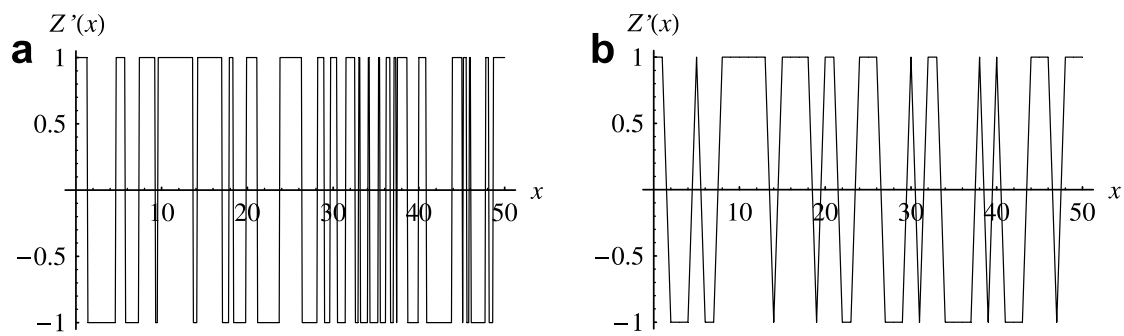

Fig. 3. (a) Approximated surface profile $Z^{\prime}(x)$ for case (5), i.e., $m / v=10$. (b) Approximated surface profile $Z^{\prime}(x)$ for the case (6), i.e., $m / v=1$. 


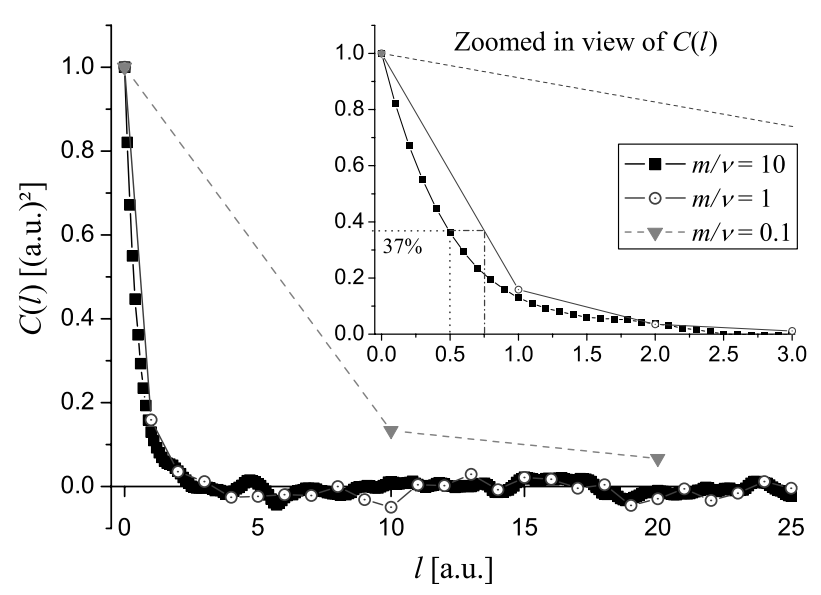

Fig. 4. Autocorrelation function $C(l)$ plotted in linear scale versus distance $l$ for the three cases (5)-(7). The full range of $l$ is plotted, which is the half of $s$ and the maximum distance of two points, so that each point has an associate partner to correlate with. Inset: Zoomed in view of $C(l)$. The correlation length $\gamma=0.5 \mathrm{UL}$ is drawn in (dotted line). If there are too few measure points one gets an artificial $\gamma^{*}$ that is too long, e.g., for $m / v=1: \gamma_{\text {lin }}^{*}=0.75 \mathrm{UL}$ (dash-dotted line), for $m / v=0.1: \gamma_{\text {lin }}^{*}=7.25 \mathrm{UL}$ (out of range).

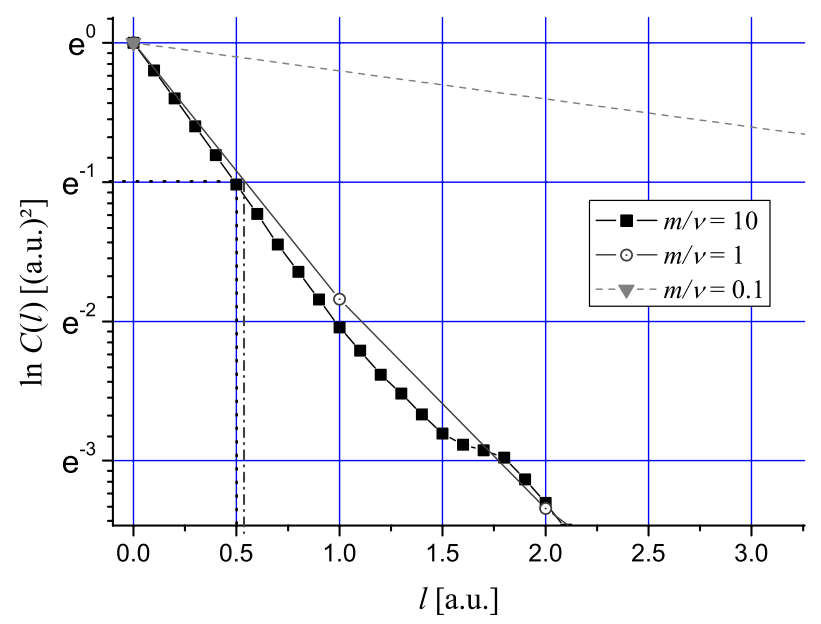

Fig. 5. Zoomed in autocorrelation function $C(l)$ (from Fig. 4) in natural logarithmic scale for the three cases (5)-(7). It is clearly visible that the beginning decay is exponential with a characteristic length $\gamma=0.5 \mathrm{UL}$ (dotted line). $\gamma$ corresponds to the negative, reciprocal slope in the natural logarithmic plot. With too big measuring steps $(m / v=1)$ the exponential fit leads to an artificial correlation length $\gamma_{\exp }^{*}=0.55 \mathrm{UL}$ (dash-dotted line). For $m / v=0.1: \gamma_{\exp }^{*}=5.0 \mathrm{UL}$ (out of range).

number of 50 points and the limited length of the profile lead to noise and oscillations given by boundary effects around zero.

The natural logarithm of the autocorrelation function can be approximated, in the interval $0 \leqslant l \leqslant 1$, as a linear function with slope -2 (see Fig. 5). This behaviour is in agreement with the analytical definition, Eq. (4), with $\gamma=0.5 \mathrm{UL}$.

For case (6) we can see that the autocorrelation function in the inset of Fig. 4 (2nd curve) has a correlation length smaller than the distance between the neighbour 'experi- mental points' (dash-dotted line). If we just connect the first two points with a straight line and take the position where this line crosses the $37 \%$ line as correlation length, we see that $\gamma$ has been shifted to higher values and, hence, has become an artificial correlation length $\gamma^{*}$. In the linear graph we get $\gamma^{*} \approx 0.75 \mathrm{UL}$. In the logarithmic graph there is a less dramatic change from $\gamma=0.5 \mathrm{UL}$ to $\gamma^{*} \approx 0.55 \mathrm{UL}$, since here a straight line contains already an exponential fit.

The results for condition (7) are shown in Fig. 4 (3rd curve). There are only three points calculated from the originally constructed profile $Z(x)$, which leads to a very high $\gamma^{*} \approx 7.25 \mathrm{UL}$ in the linear plot and $\gamma^{*} \approx 5.0 \mathrm{UL}$ in the logarithmic plot.

We observe the following tendency: if the measuring step $\Delta x$ is greater than $\gamma$, the artificial correlation length $\gamma^{*}$ increases with increasing $\Delta x$. So the intuitive condition $\Delta x<\gamma$ has been confirmed. Deducing the autocorrelation function with parameters $m / v$ between 1 and 10 and comparing the resulting correlation lengths we can give a more quantitative result: For $\Delta x<0.2 \gamma$ the deviation of the artificial correlation length $\gamma^{*}$ from the analytically determined $\gamma$ is less than $1.5 \%$, and for $0.2 \gamma<\Delta x<\gamma$ the deviation is up to approximately $6 \%$. If we remember the definitions of the parameters, we can formulate a methodic rule: To be sure to achieve a reasonable definition of $\gamma( \pm \sim 1.5 \%)$ extracted from SPM data, it is necessary to assure that

$\Delta x<0.2 \gamma \Longleftrightarrow 5$ or more datapoints in the interval $[0, \gamma]$.

If this condition is not fulfilled, one has to increase the number of sampling points, i.e., the scan resolution (decreasing $\Delta x$ ) in order to optimise the SPM measurement. This sort of quality check of SPM images could be directly integrated in the SPM control software. So it could indicate, even after only a few scanned lines, if the resolution is sufficient for a correct autocorrelation analysis.

(B): After optimisation of the analysis we can formulate a guidance for the extraction of several correlation lengths, as it is necessary for SPM imaged surfaces with non-random distribution of roughness amplitudes. The first and quite general way is the systematic analysis of STM data using monotonously increasing intervals $\Delta x$. Under the assumption of two autocorrelation lengths $\gamma_{1}$ and $\gamma_{2}$, with $\gamma_{1}<\gamma_{2}$, and the result from Section 2A the following process is quite intuitive. Starting with an interval $\Delta x_{1}<\gamma_{1}$ we know now that the autocorrelation function and $\gamma_{1}$ can be correctly determined. Increasing the interval to $\Delta x_{2}$ with $\gamma_{1}<\Delta x_{2}<\gamma_{2}$ we lose the information about the first, short autocorrelation length $\gamma_{1}$, but we are now able to extract the second, longer autocorrelation length $\gamma_{2}$. Further increasing to $\Delta x_{3}>\gamma_{2}>\gamma_{1}$ would only lead to artificial correlation lengths as shown in Section 2A.

The second possibility to analyse data with several $\gamma_{i}$ is less general, but more powerful. We fit the autocorrelation function of an STM image, using a supplemented version 
of the random telegraph function, $\widetilde{Z}(x)$, of which the autocorrelation function is deduced. For this purpose we combine the telegraph function, Eq. (3), with a sinusodial function in the following way:

$\widetilde{Z}(x)=(-1)^{n(x)}+c \sin \left(p \frac{2 \pi}{s} x\right)$,

where $c$ is the amplitude of the overlayed periodic structure, $p$ is the number of periods and we remember $s$ as the profile length. Thereby we do not get a correlation length defined by a decay of $C(l)$ to $1 / e$, but a characteristic length, which for periodical structures is also called correlation length. With Eq. (11) $\gamma_{2}$ is calculated as $s / p$.

A simpler way to extract a characteristic length is to look for local maxima of the autocorrelation function. Pronounced maxima point to a periodicity of the surface structure. This will be demonstrated and applied on series of cesium images at the end of the next chapter.

\section{STM data and their interpretation}

In this section we present STM data of several quenchcondensed cesium surfaces. These surfaces are in situ prepared in an evacuated experimental cell at low temperatures [3] (the pressure at the pump outside the cryostat is in the order of $10^{-8} \mathrm{mbar}$, but since the walls of the cell are at $4.2 \mathrm{~K}$, they act as a cryogenic pump and hence the vacuum in the cell is much less). As mentioned in the introduction Cs is, in principle, an ideal substrate for wetting studies with liquid helium [2]. Merely the surface roughness gives reason for complicating the wetting behaviour and therefore its characterisation is important [1].

Fig. 6 shows a cesium surface that was evaporated at $14.5 \mathrm{~K}$. In a chemical reaction, driven by a high current through a Cs dispenser, cesium atoms are released and deposited on HOPG or mica substrates. During the evaporation the temperature rises up to $80 \mathrm{~K}$, but afterwards cools down again to its starting value in about $30 \mathrm{~min}$.

Before analysing the topography of the imaged Cs surfaces, we have to discuss the reliability of the images themselves. SPM techniques have to be regarded as two surfaces, one of the tip and one of the sample, which are scanning each other [19]. If, for example, the curvature of the features of the sample surface is smaller than the curvature of the tip, the scanned image at this position will be dominated by the shape of the tip. We believe that this effect is negligible in the STM image shown in Fig. 6, since the tip is able to resolve the small valleys between the islands, which have a width of approximately $10 \mathrm{~nm}$ (so features below $10 \mathrm{~nm}$ could hardly be imaged). In our case most features have sizes of the order of $40-60 \mathrm{~nm}$ and should therefore be quite well reproduced.

The histogram of the roughness distribution of this cesium surface (Fig. 6) is presented in the inset of Fig. 7. The data are fitted with the statistical defined $W(Z)$, see Eq. (1), here in the form of a Gaussian distribution, i.e.,

$W(Z)=\frac{1}{\left(2 \pi \Delta^{2}\right)^{1 / 2}} \exp \left(-\frac{\left(Z-Z_{\mathrm{os}}\right)^{2}}{2 \Delta^{2}}\right)$

where the standard deviation $\Delta$ is equal to $2.09 \mathrm{~nm} . Z_{\mathrm{os}}$ is an horizontal offset value, which exists because we set, for an uniformly treatment of the images, the mean value of all heights to zero (instead to set the value with the highest frequency to zero as it is done often).

The histogram gives only information about the frequency of the different heights that appear in the image. The fact that the Gaussian distribution fits quite well argues for a random distribution of roughness of this cesium surface. We will compare this result with the correlation properties below.

The autocorrelation function of this surface can be extracted from the single scan lines $\left(\widehat{=} Z^{\prime}(x)\right)$ of Fig. 6 by using Eqs. (8) and (9) and subsequent averaging. Fig. 7 shows the corresponding $C(l)$ of Fig. 6. Also drawn in is the correlation length where the correlation decays to roughly $37 \%$ (i.e., $1 / e$ ). An evaluation of the image perpendicular to the scan direction has to be considered with caution. If the STM image has not a perfect quality, artefact lines could noticeably distort the autocorrelation function. During averaging in scan direction such a line can be considered as negligible, but in the perpendicular direction
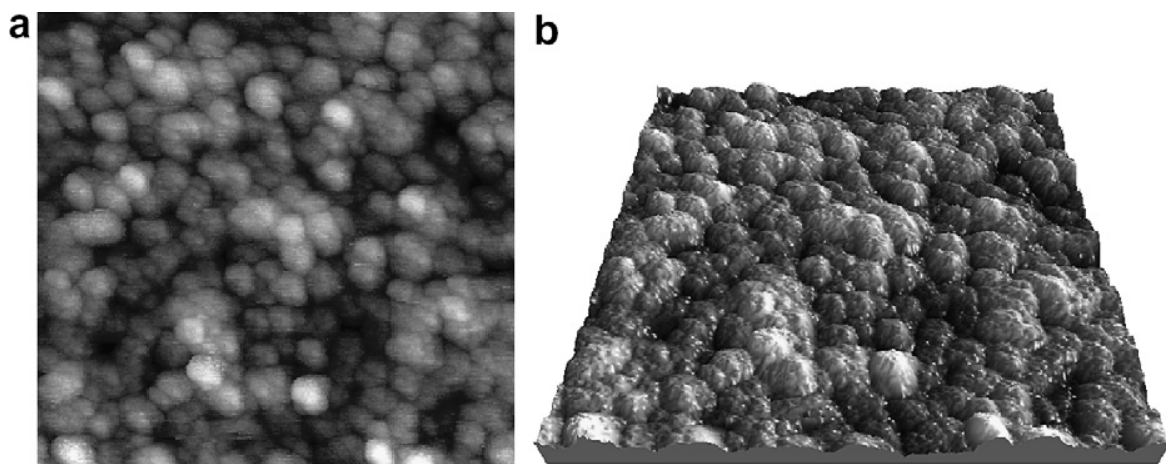

Fig. 6. (a) STM image with a side length of $s=660 \mathrm{~nm}$ of cesium evaporated on HOPG at $14.5 \mathrm{~K}$ (heated up to $80 \mathrm{~K}$ during evaporation). The range of height is $22.1 \mathrm{~nm}$ (from black to white). Tunneling current: $40 \mathrm{nA}$, tunneling bias: $180 \mathrm{mV}$. (b) 3D view of (a). The scale of height is three times inflated for a better view of the structure of the surface. 


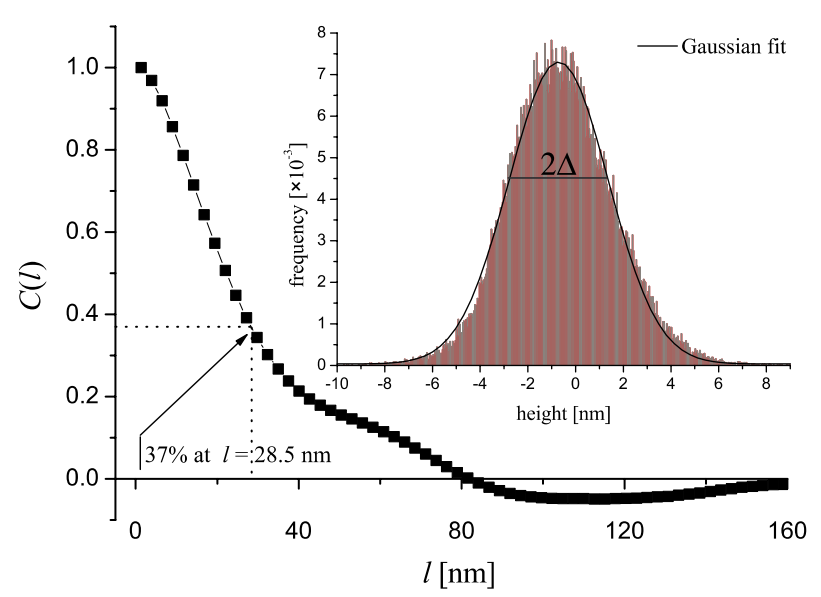

Fig. 7. Normalised autocorrelation function of the cesium surface shown in Fig. 6. The curve corresponds to the averaged autocorrelation function of the lines in scan direction. Inset: Histogram of the Cs surface shown in Fig. 6. The Gaussian fit has an $x$-offset of $Z_{\mathrm{os}}=-0.71 \mathrm{~nm}$ and the half of its width is equal to $\Delta$ (色standard deviation), which is $2.09 \mathrm{~nm}$.

there would be a wrong value in all lines. So we just discuss the evaluation of STM images in scan direction.

In order to apply the results of Section 2 we have to compare the measuring step with the correlation length, see Eq. (10). To demonstrate this, we count the measuring points in the plot of the autocorrelation function (Fig. 7) from zero to the drawn-in correlation length (dotted line). If this number is around 5 or higher, the condition (10) is fulfilled. Otherwise we have to increase the scan resolution, i.e., to decrease $\Delta x$.

Furthermore, we see that the amplitude of $\sqrt{\left.C(l)\right|_{l \rightarrow 0}}=\sqrt{6.19 \mathrm{~nm}^{2}}=2.49 \mathrm{~nm}$ and the standard deviation in $W(Z), \Delta=2.09 \mathrm{~nm}$ (see inset in Fig. 7), are comparable. According to Eq. (2) this should be the case for a random roughness distribution.

A random roughness, in the way it occurs in the random telegraph function, has an exponential decay behaviour in $C(l)$. This corresponds to a description with only one characteristic length $\gamma$. Real cesium surfaces, however, show a subsequent increase in the correlation, which indicates that the surface image contains a periodicity. This suits to the roughly periodic island structure, visible in Fig. 6. Therefore it can be described with a second correlation length $\gamma_{2}$, and if the correlation length of the smallest measurable structure is $\gamma_{1}$, we get $\gamma_{1}<\gamma_{2}$. The overlayed structure on a larger length scale is also responsible for the appearance of negative correlation values.

Now we want to extract $\gamma_{2}$. The first way of extracting correlation lengths of higher order, as described in Section $2 \mathrm{~B}$, is in our case not applicable. Indeed $\gamma_{1}$ and $\gamma_{2}$ are sufficiently separated in length to distinguish from each other, as we saw above. But if we increase the sampling width $\Delta x$ in such a way that $\gamma_{1}$ can not be extracted anymore, there are too few points to extract $\gamma_{2}$ without becoming artificial. Such a situation is demonstrated in Section 2A, i.e., the scanned fraction of the surface is too small to have enough sample points in the profile at this lower resolution.

So, we try to fit the details of such behaviour of $C(l)$ with the supplemented telegraph function, see Eq. (11), as announced in Section 2B. The sinusodial function, added to Eq. (3), with a certain period and amplitude inserts a second correlation, in this case a periodic one. For $p=1.9$ and $c=0.33$ the generated surface profile is shown in Fig. $8 \mathrm{a}$ and its autocorrelation function in Fig. 8b. This leads to the same qualitative development of the autocorrelation function as in Fig. 7. So we conclude that the surface in Fig. 6 has an additional periodic correlation of the length: $\gamma_{2} \approx s / p=350 \mathrm{~nm}$, where we remember that $s$ is the profile length, in this case $s=660 \mathrm{~nm}$. Though $\gamma_{2}$ is rather large, it seems reasonable as even the bare eye can recognise a superordinated structure. In the lower half of Fig. 6 we see two holes that cover several of the small islands. This corresponds to a characteristic length about half of $s$.

Although the histogram, see inset of Fig. 7, indicates a random distribution of roughness for the data shown in Fig. 6, we get a different information from the correlation analysis. The deviation of $C(l)$ from a delta function and even from the standard exponential behaviour, together with the existence of a second correlation length, argue against a normal random roughness. This confirms the usefulness of correlation analysis in addition to the usual histogram, see also Ref. [14].

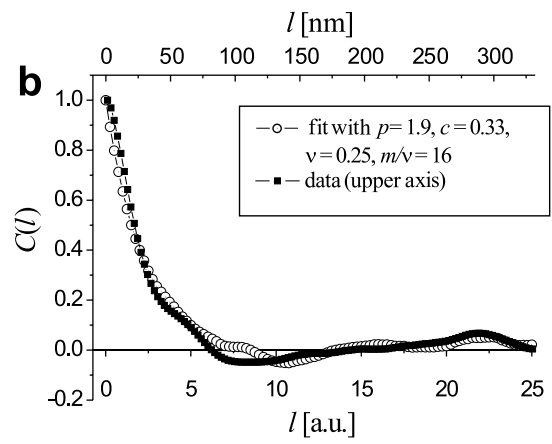

Fig. 8. (a) An example of a telegraph function with an added sinusodial function with the parameters $p=1.9, c=0.33$, and $v=0.25$. This results in a profile with a decay correlation length and a further correlation length on a different length scale. (b) Averaged and normalised autocorrelation function of 50 realisations of a profile as shown in (a) versus distance (open circles). This serves as a qualitative fit for the autocorrelation function in Fig. 7 (solid squares). 
Different preparation methods lead to different profiles of cesium surfaces. For example, a higher starting temperature leads to a smoother surface (Fig. 9) with a longer correlation length (Fig. 10: $\gamma=175 \mathrm{~nm}$ ). For this surface the histogram, inset of Fig. 10, cannot be well described by a Gaussian function, since it has an asymmetric shape. Therefore, the corresponding $\Delta=3.83 \mathrm{~nm}$ is somewhat less similar to $\sqrt{\left.C(l)\right|_{\rightarrow 0}}=\sqrt{50.27 \mathrm{~nm}^{2}}=7.09 \mathrm{~nm}$.

Similar to Fig. $8 \mathrm{~b}$ we apply the same sort of fit to the autocorrelation function deduced from the image shown in Fig. 9. As this surface has much longer correlation lengths, the parameters of supplemented telegraph function have to be more strongly modified, see Fig. 11. Hence, a periodicity of $p=1.45$ gives a good fit and leads to a second correlation length of $\gamma_{2} \approx s / p=915 \mathrm{~nm}$. In this case $\gamma_{2}$ is more than $2 / 3$ of $s$ and its illustrative interpretation is more difficult than the result of the image shown in Fig. 6. The surface shown in Fig. 9 seems smoother, but has a big protrusion at the upper side of the image. This could explain that $\gamma_{2}$ is almost comparable with $s$. Thus for a more reliable extraction of the second order correlation of this structure an image of a larger section of the surface would have been of advantage, however, this is not possible with our current setup.

A further application of analysing details of the autocorrelation function is described in the following by looking at the changes of two series of cesium surfaces. Fig. 12 shows the first and the third image of a series of three images, which was taken one after the other at exactly the same position and at the same temperature. The time between the two images is about $57 \mathrm{~min}$. There are indications of a double tip as we find many pairs of features with similar distance $(\approx 28 \mathrm{~nm})$ and angle $\left(\approx-15^{\circ}\right)$. However, a few double structures are also visible under quite different angles. In addition, there are also single features of bigger size but of round shape. These facts do not fit to the apparent double tip.

The visible changes in the surface structure are accompanied by changes of the first correlation length $\gamma_{1}$ as well

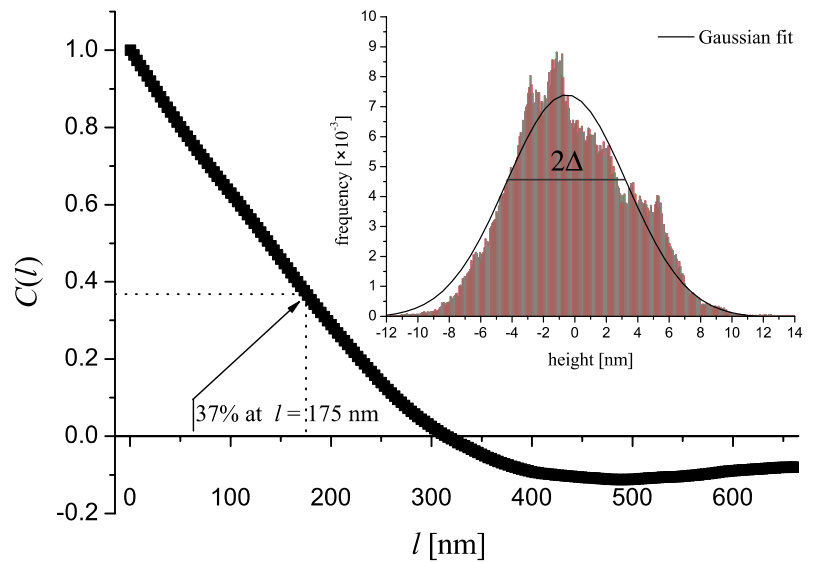

Fig. 10. Normalised autocorrelation function of the cesium surface shown in Fig. 9. Inset: Histogram of the Cs surface shown in Fig. 9. The Gaussian fit has an $x$-offset of $Z_{\mathrm{os}}=-0.53 \mathrm{~nm}$ and the half of the width is equal to $\Delta$ (气standard deviation), which is $3.83 \mathrm{~nm}$.

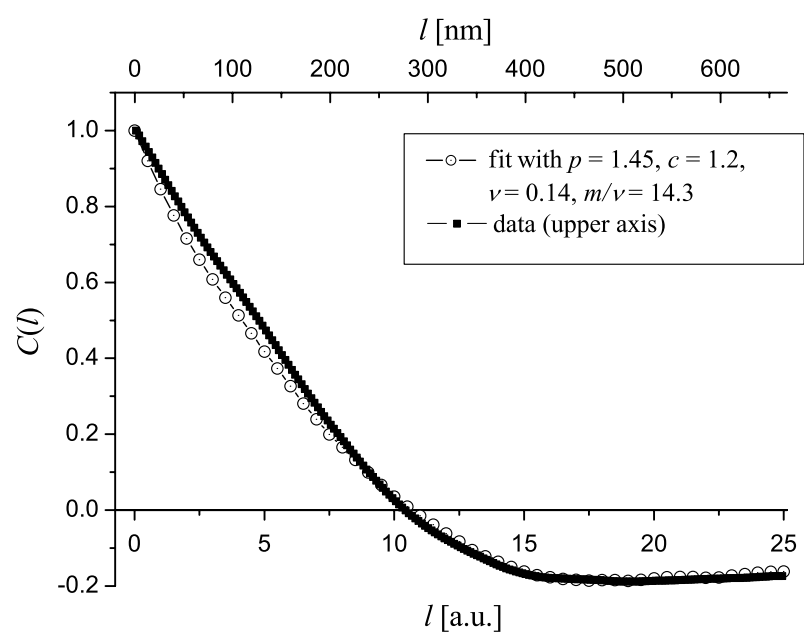

Fig. 11. Averaged and normalised autocorrelation function of 50 profiles, created with the parameters $p=1.45, c=1.2, v=0.14$ and $m / v=14.29$, versus distance (open circles). This serves as a qualitative fit for the autocorrelation function corresponding to the cesium surface shown in Fig. 9 (solid squares).
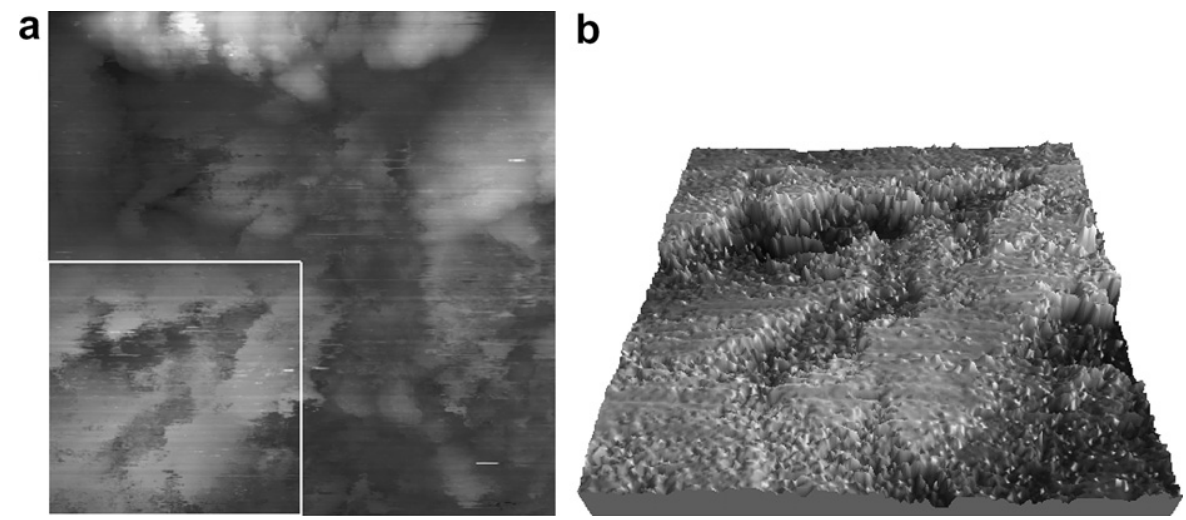

Fig. 9. (a) STM image with a side length of $s=1330 \mathrm{~nm}$ of cesium evaporated on HOPG at $85 \mathrm{~K}$ (heated up to $135 \mathrm{~K}$ during evaporation). The range of height is $34.8 \mathrm{~nm}$ (from black to white). Tunneling current: $4 \mathrm{nA}$, tunneling bias: $100 \mathrm{mV}$. (b) 3D view of the section indicated in (a), equal in size to Fig. $6 \mathrm{~b}$. The scale of height is three times inflated. 
a

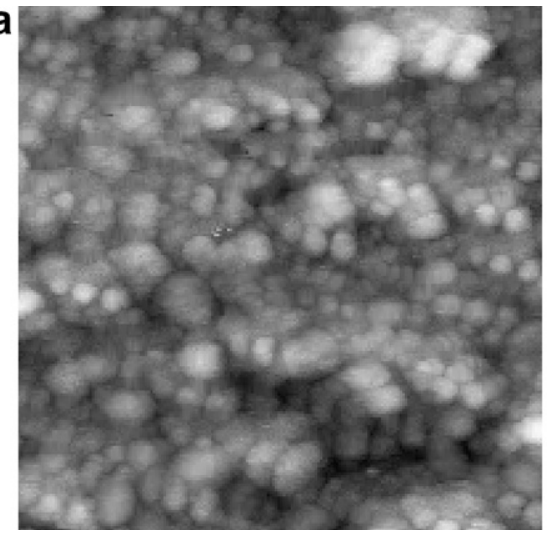

b

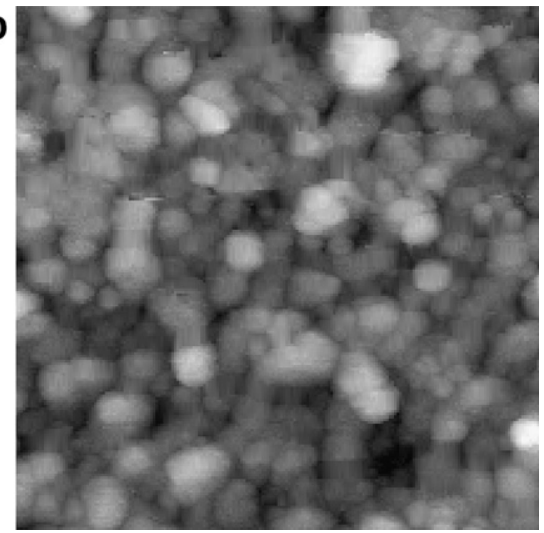

Fig. 12. (a) First image of a series of three cesium surfaces with a side length of $464 \mathrm{~nm}$ of cesium (same substrate as in Fig. 6). The range of height is $14.7 \mathrm{~nm}$ (from black to white). (b) Third image of the series described in (a). The range of height is $17.3 \mathrm{~nm}$. Both images: tunneling current: $40 \mathrm{nA}$, tunneling bias: $180 \mathrm{mV}, T=14.5 \mathrm{~K}$. The time between the two images is about $57 \mathrm{~min}$.

Table 1

The first correlation lengths $\gamma_{1}$ and the positions of the first local maxima of the autocorrelation function, which correspond to the second correlation lengths $\gamma_{2}$, of two series of cesium images

\begin{tabular}{|c|c|c|c|c|}
\hline & \multicolumn{2}{|c|}{ Series no. 1 (side length 464 nm, Fig. 12) } & \multicolumn{2}{|c|}{ Series no. 2 (side length $597 \mathrm{~nm}$, not shown) } \\
\hline & $\gamma_{1}(\mathrm{~nm})$ & 1 st $\max . \equiv \gamma_{2}(\mathrm{~nm})$ & $\gamma_{1}(\mathrm{~nm})$ & 1 st $\max . \equiv \gamma_{2}(\mathrm{~nm})$ \\
\hline 1st image & $28.6 \pm 0.70$ & $76 \pm 1.17$ & $26.8 \pm 2.3$ & $98 \pm 6.1$ \\
\hline 3rd image & $20.1 \pm 0.60$ & $80 \pm 1.83$ & & \\
\hline
\end{tabular}

The first series is shown in Fig. 12 (only first and third image), and its autocorrelation functions are plotted in Fig. 13. The second series is not shown.

as shifts of the position of local maxima of the autocorrelation function, which corresponds to the second correlation length $\gamma_{2}$. So here we use the simpler way of extracting periodic lengths, as described in Section 2B. A summary of the positions of the maxima averaged from forward and backward scan of the corresponding image is given in Table 1. The two images of the second series are not shown (they were taken in the same experimental run with similar parameters). In both series the positions of the local maxima are shifted to higher values. In Fig. 13 we see that the maxima are getting more pro-

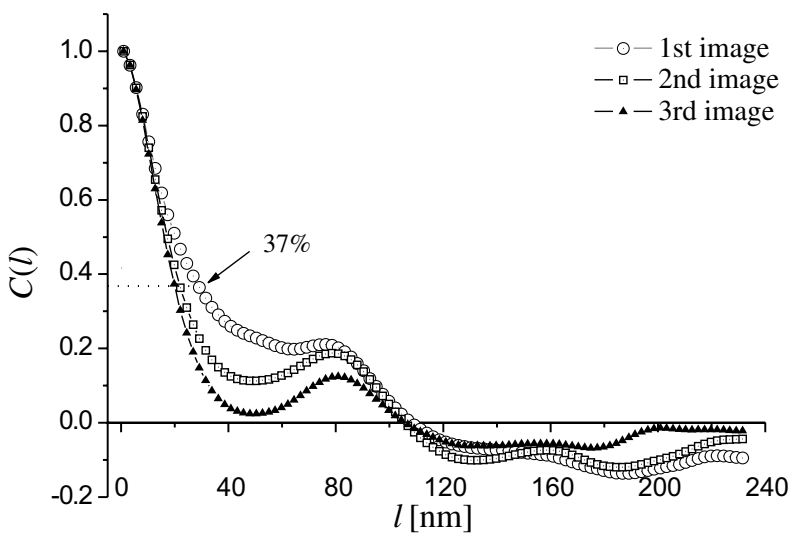

Fig. 13. Normalised autocorrelation function of a sequence of cesium surface images. The first and the third curve corresponds to Fig. 12a and $\mathrm{b}$, respectively (the 2 nd image is not shown). The deduced correlation lengths (where $C(l)$ decayed to $\approx 37 \%$ ) are listed in Table 1 . nounced. And therewith $\gamma_{1}$ is getting shorter, since the decay gets steeper. The maxima of the second series are not so clearly developed. Here $\gamma_{1}$ increases slightly.

When there are changes in STM images, the standard suspicion is a change of the shape of the tip. At first sight it looks as if the image shown in Fig. 12a bases on a double tip and the image shown in Fig. 12b does not. A closer look reveals that most of the apparent double features also exist in Fig. 12b, but with less contrast. However, the bigger islands gained contrast and so seem to have risen. This is in agreement with the increased range of height from $14.7 \mathrm{~nm}$ to $17.3 \mathrm{~nm}$. As in many places in Fig. 12b the small features are still visible, an increased curvature of the tip seems not to be responsible for the differences between Fig. 12a and b.

A possible reason for the changes of structure could be the high average tunneling current of $40 \mathrm{nA}$ and the shape of the time variation of the current. It contains many sharp needles of high values of current with very low values in between. This sort of tunneling current could have induced energy into the surface and excite some changes of its structure [3].

\section{Conclusion}

Surface roughness is an omnipresent property of most solid substrates. It plays an important role in many physical research activities, i.e., the wetting behaviour of liquid helium on a cesium substrate. For this purpose we gained access to the surfaces of quench-condensed Cs films which 
we analysed regarding their roughness properties. For our analysis we have chosen two basic characteristics of roughness, i.e., the distribution function of roughness amplitudes (i.e., histogram) and the autocorrelation function. We showed a way how to assure that the extraction of the autocorrelation function from a scanning probe microscopy image is correct, so that the correlation length, where the function decays to $1 / e$, is not artificially increased. This was supported by a numerical 'experiment' with a random telegraph function, which was simulatively scanned with different scan resolutions. The resulting requirement was that the correlation length should be at least five times larger than the probing step.

Often SPM imaged surfaces contain more than one correlation length. We presented some methods of how to extract such further correlation lengths of higher order from the data. With STM measurements of quench-condensed cesium films as example we discussed and compared the two basic roughness properties and applied, as far as possible, the above mentioned methods. We saw that the information of the histogram does not contain all characteristics of the surface structure. It does not indicate the deviation of a random distribution of roughness amplitudes, whereas the autocorrelation function reveals more details like higher orders of correlation. We think that these characteristic lengths will play a role in further statistical calculation on the influence of surface roughness on the wetting behaviour of liquid helium on cesium surfaces.

\section{Acknowledgement}

We would like to thank E. Rolley for helpful discussions. This work has been supported by the 'Deutsche Forschungsgemeinschaft' under grant KL 1186/3.

\section{References}

[1] J. Klier, P. Leiderer, D. Reinelt, A.F.G. Wyatt, Phys. Rev. B 72 (2005) 245410, and references therein.

[2] E. Cheng, M.W. Cole, J. Dupont-Roc, W.F. Saam, Rev. Mod. Phys. 65 (1993) 557.

[3] M. Zech, A. Fubel, P. Leiderer, J. Klier, J. Low Temp. Phys. 137 (2004) 179

[4] E.S. Sabisky, C.H. Anderson, Phys. Rev. A 7 (1973) 790.

[5] S.M.M. Ramos, E. Charlaix, A. Benyagoub, Surf. Sci. 540 (2003) 355.

[6] Y. Zhu, S. Granick, Phys. Rev. Lett. 88 (2002) 106102.

[7] R.R. Netz, D. Andelman, Phys. Rev. E 55 (1996) 687.

[8] J. Klier, M. Zech, A. Fubel, P. Leiderer, V. Shikin, J. Low Temp. Phys. 138 (2005) 355.

[9] S. Mora, J. Daillant, K. Mecke, Phys. Rev. Lett. 90 (2003) 216101.

[10] D. Cieslikowski, P. Leiderer, A. Dahm, Can. J. Phys. 65 (1987) 1525.

[11] J. Klier, A. Würl, P. Leiderer, G. Mistura, V. Shikin, Phys. Rev. B 65 (2002) 165428

[12] D. Coimbra, S. Sokolov, J. Rino, N. Studart, J. Low Temp. Phys. 126 (2002) 505.

[13] J. Vorberg, S. Herminghaus, K. Mecke, Phys. Rev. Lett. 87 (2001) 196105.

[14] P. Dooley, S.L. Bernasek, Surf. Sci. 406 (1998) 206.

[15] E.A. Eklund, E.J. Snyder, R.S. Williams, Surf. Sci. 285 (1993) 157

[16] G. Rasigni, F. Varnier, M. Rasigni, J.P. Palmari, A. Llebaria, Phys Rev. B 25 (1982) 2315.

[17] A.T. Bharucha-Reid, Elements of the Theory of Markov Processes and Their Applications, McGraw-Hill, New York, 1960.

[18] V.I. Tikhonov, M.A. Mironov, Markovskie prozessi, Moskva, Sov. Radio, 1977.

[19] E.J. van Loenen, D. Dijkkamp, A.J. Hoeven, J.M. Lenssinck, J. Dieleman, Appl. Phys. Lett. 56 (1990) 1755. 